\title{
Evidence for a C14 Frank-Kasper Phase in One-Size Gold Nanoparticle Superlattices
}

\author{
Stéphanie Hajiw, Brigitte Pansu, ${ }^{*}$ and Jean-François Sadoc \\ Laboratoire de Physique des Solides, Bât 510, UMR-CNRS 8502, Université Paris-Sud, \\ 91405 ORSAY Cedex, FRANCE \\ E-mail: brigitte.pansu@u-psud.fr
}

\begin{abstract}
The Frank-Kasper phases were already known in 1982 when quasicrystals have been discovered but their complex architectures are now considered as making a link between simple close-packed periodic structures and some quasiperiodic ones. These tetrahedrally close packed structures are observed in many materials from elements to intermetallics as well as self-assembled soft materials like micellar systems, dendritric liquid crystals, star polymers and more recently block-copolymers or heated gold nanocrystal superlattices. We report here the existence of a Frank-Kasper phase with hexagonal symmetry $\left(\mathrm{MgZn}_{2}\right.$ type, also labelled $\left.\mathrm{C} 14\right)$ in superlattices of monodisperse hydrophobically-coated gold particles at room temperature obtained from suspensions in various solvents. The existence of such a structure in this system is analysed in terms of geometrical parameters including gold core diameter, ligand length and grafting density and an energetic approach based on van der Waals attraction. Hydrophobically-coated gold nanoparticles is a new system that exhibit a Frank and Kasper phase built by one-size objects. This result opens a route towards nanoparticle superlattice with complex structures and thus original physical properties.
\end{abstract}

${ }^{*}$ To whom correspondence should be addressed 


\section{Keywords}

Nanocrystals; superlattices; colloids; self-assembly

Understanding how atoms or particles can fill the space is a very old challenge. When particles or atoms behave like hard spheres, the structures that they form are compact structures : face centered cubic (FCC) or hexagonal compact (HCP). Other structures like body-centered cubic structure (BCC) or Frank-Kasper phases (FK) require more complex interactions $^{1}$ or anisotropic shapes. ${ }^{2}$ Sixty years ago, Frank and Kasper ${ }^{3}$ have investigated many complex alloy structures, constructing packings of polyhedra with large coordination number $(\mathrm{Z}=12,14,15$ or 16$)$ in order to maintain tetrahedral close packing. After the discovery of quasicrystals,${ }^{4}$ it was recognized that the Frank-Kasper phases complex architectures were the bridging between classical close-packed periodic structures and some quasiperiodic ones. They thus regain much attention in order to understand how to get quasicrystalline materials. In the Frank-Kasper (FK) structures, there are at least two types of sites with different environments. This explains why, in atomic systems, these structures are mainly observed in alloys with at least two types of atoms, with some exceptions such as $\beta$-Tungsten (A15 phase) or $\beta$-Uranium ( $\sigma$ phase). Since soft particles can adapt their shape to the local geometrical constraints, they are excellent systems to search for FK phases and thus quasicrystalline structures. ${ }^{5,6}$ In these systems, beyond thermodynamic interaction, entropy is suspected to drive new principles of self-organization ${ }^{7,8}$ and FCC, HCP and BCC structures but also FK phases are expected as for atomic systems. Frank-Kasper phases have already been found in micellar systems, ${ }^{9}$ liquid crystals, ${ }^{5,10}$ star polymers, block-copolymers ${ }^{11,12}$ and very recently in heated gold nanocrystal superlattices. ${ }^{13}$ Micellar systems ${ }^{9}$ exhibit two different FK phases: the A15 phase for direct micelles and the C15 phase for inverted micelles (Figure 1). The A15 phase is a cubic phase with Pm3n as symmetry group, a $\mathrm{AB}_{3}$ stoichiometry, 8 spheres per cubic cell and $Z=12$ (icosahedron) or $Z=14$ coordination numbers. The C15 Laves phase ${ }^{14}$ is also cubic with Fd3m as space group, a $\mathrm{AB}_{2}$ stoichiometry, 24 spheres per cubic cell and $\mathrm{Z}=16$ or $\mathrm{Z}=12$ coordination numbers. In dendrimers ${ }^{10}$ as in polymeric sys- 
tems, ${ }^{11,12}$ a $\sigma$ FK phase with tetragonal symmetry $\left(\mathrm{P} 4_{2} / \mathrm{mnm}\right)$ has also been reported. With 30 spheres per unit cell, it is a dodecagonal quasicrystal approximant structure primarily reported in numerous metal alloys. However, in surfactant or block-copolymer self-assembly, the micelles are not identical and the system is thus closer to alloy structure. For gold nanoparticles, the formation of the FK phases was linked to the growth of some particles upon heating leading to several gold core sizes. Only the dendrimers can be really considered as monodisperse objects building FK phases and dodecagonal quasicrystals. Nevertheless establishing links between all these systems is essential to improve understanding of complex crystallization. This is clearly an important challenge in order to grow materials based on metallic nanoparticles with complex symmetries and thus original physical properties.

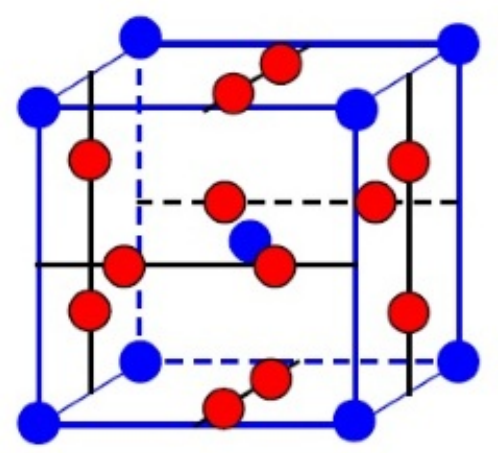

a)

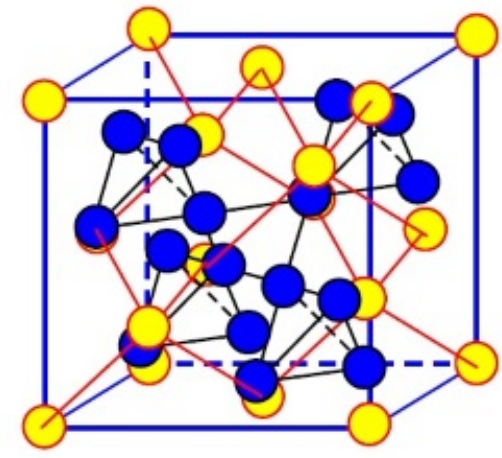

b)

Figure 1: a) A15 Frank-Kasper structure with space groupe Pm3n (Q223) and $\mathrm{AB}_{3}$ stoichiometry b) C15 Frank-Kasper structure with space group Fd3m (Q227) and $\mathrm{AB}_{2}$ stoichiometry.

Metallic or semi-conducting nanoparticles (NP) surrounded by grafted ligands forming a soft corona around them are good candidates as soft particles. Moreover they build superlattices very easily. For this reason, the packing of gold nanoparticles capped with alkanethiols has been extensively investigated. Until now only FCC or BCC structures have been observed at room temperature. But others structures and notably quasicrystalline ones could offer interesting physical properties. One pertinent parameter that drives the transition from FCC to BCC structure is the ratio $x=L / R$ where $L$ is the ligand extended length and $R$ the 
radius of the solid core. When $x$ is smaller than typically 0.7 , the FCC packing is favored. ${ }^{15,16}$ For large $x, \mathrm{BCC}$ or BCT (deformed BCC) packings can be observed. Soft nanoparticle superlattices are usually nucleated through the evaporation of the suspending solvant and the nucleation kinetics is also essential in controlling the final structure. ${ }^{17}$ Recent experiments have shown that superlattices of dodecanethiol-capped $1.8 \mathrm{~nm}$ diameter Au nanocrystals $(x$ close to 2) can undergo a non-reversible series of ordered structure transitions at high temperature. ${ }^{13}$ Among these phases, several complex pseudo-FK phases have been observed: the cubic $\mathrm{NaZn}_{13}$-type structure (Fm3c), the hexagonal $\mathrm{CaCu}_{5}$-type structure $(\mathrm{P} 6 / \mathrm{mmm})$. But in this experiment, the structure change is associated to the NP growth and even coalescence leading to various sizes of nanoparticles.

\section{Results and discussion}

\section{Experimentals Results}

Whatever the crystallization process used and after a certain time, all samples show the same typical SAXS pattern. It is clearly not cubic but compatible with a compact-hexagonal type structure with the first peak located close to $0.12 \AA^{-1}$ and thus a lattice parameter $a \approx 6.5$ $\mathrm{nm} c=\sqrt{8 / 3} a \approx 10.5 \mathrm{~nm}$. The lattice parameter $a$ is always close to twice the distance $D_{P}$ for which the ligands chains are fully interpenetrated. This large cell parameter is very reminiscent of what has been observed ${ }^{13}$ on suspension of dodecanethiol-coated gold nanoparticles at high temperature but the authors have attributed the large lattice constant to the NP's growth. Figure 2 shows a diffraction pattern that can be described by a hexagonal lattice with a large cell parameter. The first peaks are less intense. Some peaks close to $q=0.2 \AA^{-1}$ are more intense. This clearly shows that, in the large cell, the crystallographic motif is rather complex. In emulsions, the observed peaks are larger: the domains, confined in droplets, are smaller and the lattice parameter is less well defined, it could also be not perfectly homogenous. Nevertheless this parameter lies in the same range as for crystals 
built in capillaries.

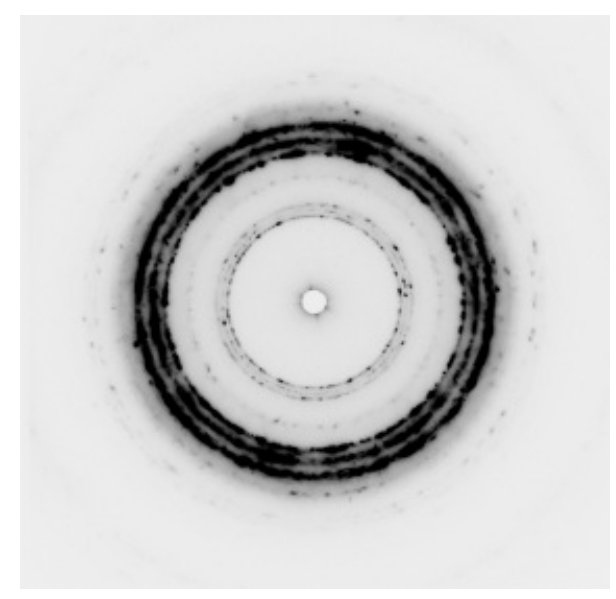

a)

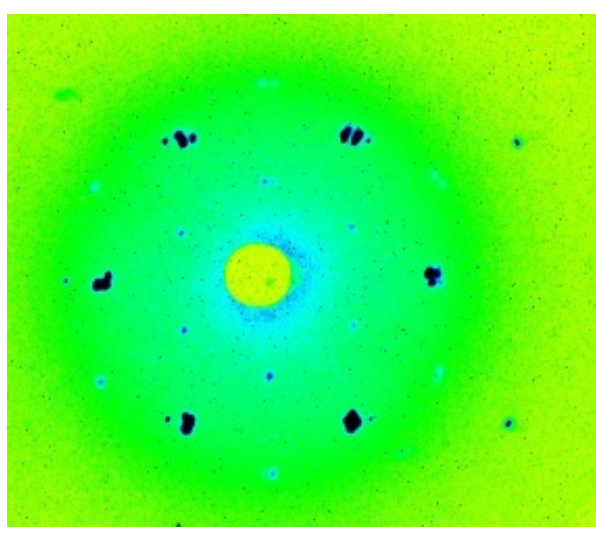

b)

Figure 2: a) Textured pattern obtained by SAXS on gold NP superlattices, b) Diffraction pattern on a quasi single domain showing a 3 -fold axis.

To confirm the large cell parameter, cryo-TEM experiments have been performed on superlattices built by Oswald ripening of NP suspension-in-water emulsions. Using this method, small crystals stabilized by surfactant can be grown and observed by cryo-TEM . X-ray scattering on these emulsions also shows Bragg peaks around $0.108 \AA^{-1}$ even if the peaks are large due to the small grain size but perhaps also to a polydispersity in the lattice parameters. Some images as Figure 3a confirm that the lattice parameter is typically twice larger than the particle diameter. Indeed Figure 3a shows a hexagonal pattern with typical distances $6.4-6.7 \mathrm{~nm}$. Figure 3b exhibits layers with a distance $d \sim 3.3 \mathrm{~nm}$. CryoTEM expriments also show that without heating the nanoparticle core size is constant. The sulphur-gold bond is also very stable and thus, at room temperature, the number of ligands per gold core can reasonably be considered as constant. Thus the system contrary to what is observed in ${ }^{13}$ really remains monodisperse.

\section{Determination of the structure}

X-ray scattering experiments clearly shows that the structure is hexagonal but with a parameter cell twice larger than expected for single spheres. Therefore more complex architec- 


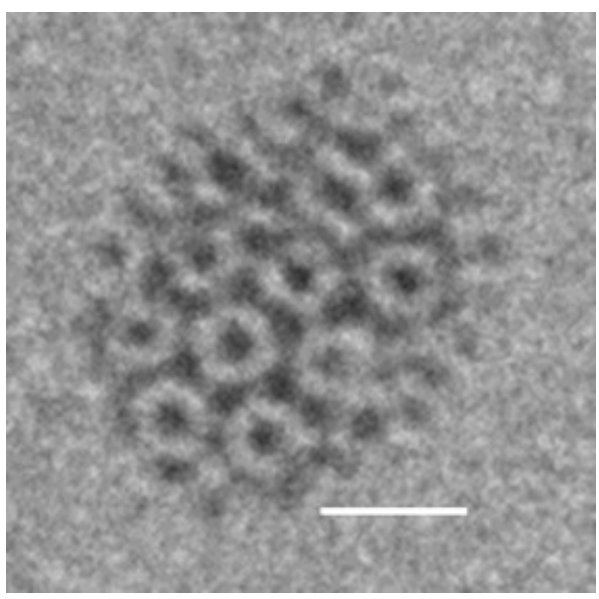

a)

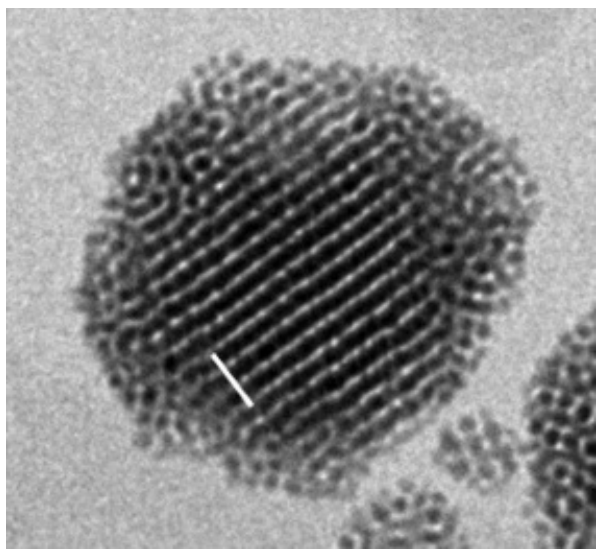

b)

Figure 3: Cryo-TEM image of small grains of hydrophobically-coated gold NP superlattices grown by Ostwald ripening of a doped oil in water emulsion (white scale bar $10 \mathrm{~nm}$ ). The mean NP diameter determined by SAXS is $2.5 \mathrm{~nm}$. a) Distance between the cell centers: 6.3 to $6.7 \mathrm{~nm} \mathrm{b)} \mathrm{The} \mathrm{observed} \mathrm{period} \mathrm{between} \mathrm{the} \mathrm{lines} \mathrm{is} 3.3 \mathrm{~nm}$.

tures as those of FK or pseudo FK structures have naturally been considered. Several FK structures are compatible with the cryo-TEM images: the C14 structure or $\mathrm{MgZn}_{2}$ structure (Figure 4a) and the $\mathrm{Z}$ structure or $\mathrm{Zr}_{4} \mathrm{Al}_{3}$ structure (Figure $4 \mathrm{~b}$ ). Other FK phases or pseudo-FK phases exhibiting hexagonal symmetry contain a greater number of spheres in the cell. Their cell parameter would be too large compared to the experimental observations and they thus have been rejected.

In the $\mathrm{C} 14$ structure (SI1), the Zn atoms are arranged in parallel hexagonal layers: $h_{1}$ at $\mathrm{z}=0$ and $h_{2}$ at $\mathrm{z}=1 / 2$ or in Kagome layers $K_{1}$ at $\mathrm{z}=1 / 4$ and $K_{2}$ at $\mathrm{z}=3 / 4$. Each Zn Kagome layer is sandwitched between two hexagonal layers made by $\mathrm{Mg}$ atoms as for instance $H_{1^{-}}$ $K_{1}-H_{1}^{\prime}$. The hexagonal cell thus contains $8 \mathrm{Zn}$ atoms and $4 \mathrm{Mg}$ atoms. For identical hard spheres with diameter $D$, the cell parameter would be $a=2 D$ and the volume fraction $55 \%$. This structure is not really compact and, as for the other FK phases, the local environments play a large role. There are three equivalent positions with different environments (SI2). The coordination number $\mathrm{Z}$ is $\mathrm{Z}=16(\mathrm{Mg})$ and $\mathrm{Z}=12(\mathrm{Zn})$. For the $\mathrm{Zn}$ sites, the coordination polyhedra are thus deformed icosahedra. The Voronoi cell volume around each atom is $0.13432 a^{3}$ for the $4 \mathrm{Mg}$ sites, $0.11025 a^{3}$ for the $2 \mathrm{Zn}$ sites (h-type), $0.1094 a^{3}$ for the $6 \mathrm{Zn}$ 
sites (K-type) for a cell volume equal to $\sqrt{2} a^{3}$. Each Voronoi cell is thus linked to a unique gold core but can be filled by part of corona grafted on different gold cores.

In the $\mathrm{Z}$ structure, the $\mathrm{Al}$ atoms are stacked in Kagome-mesh layers at $\mathrm{z}=0$, half $\mathrm{Zr}$ are situated in a hexagonal mesh at $\mathrm{z}=1 / 4$ and $\mathrm{z}=3 / 4$. The other half $\mathrm{Zr}$ are stacked in layers at $\mathrm{z}=0.5$ and are situated just above the $\mathrm{Al}$ triangles of the Kagome layers. For identical hard spheres with diameter $D$, the cell parameters would be $c=2 D \approx a$ and the volume fraction $55 \%$. The $\mathrm{Al}$ coordination number is 12 . The $\mathrm{Zn}$ coordination number is either 14 or 15 and the mean coordination number is 13.43 .

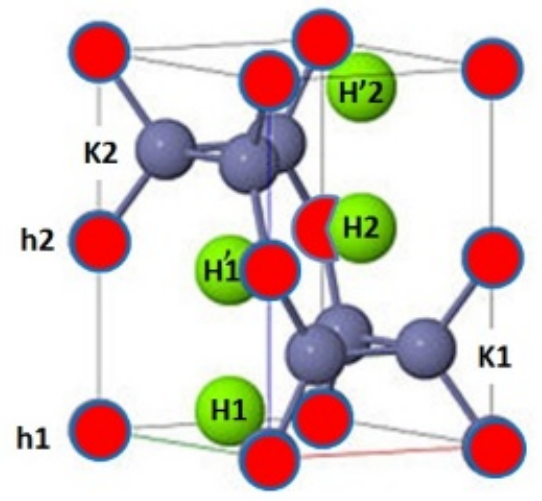

a)

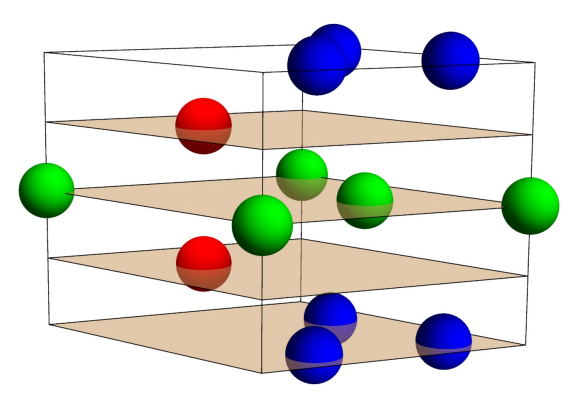

b)

Figure 4: Hexagonal Frank-Kasper phase a) the $\mathrm{MgZn}_{2}$ phase also labelled $\mathrm{C} 14$ (P6 $\left.3 / \mathrm{mmc}\right)$ $\mathrm{c} / \mathrm{a}=\sqrt{\frac{3}{8}} \quad$ b) the $\mathrm{Zr}_{4} \mathrm{Al}_{3}$ phase, also labelled $\mathrm{Z}(\mathrm{P} 6 / \mathrm{mmm}), \mathrm{c} / \mathrm{a}=0.992$

In the NP superlattices, each Voronoi cell is occupied by a nanoparticle, that is a gold core surrounded by the soft ligands and different sites correspond to different conformation of the ligands. The scattered intensity is dominated by the contribution of the gold cores and the chains cannot be detected. The form factor of the NPs is thus only that of the gold core determined by SAXS on dilute suspensions. The expected intensity can easily be computed for powdered pattern (SI3) and compared with the radial intensity measured on diffraction pattern as that of Figure 2a. Superlattices are in contact with fluid suspension and the total intensity has been analysed as the sum of the intensity scattered by a crystalline powder (first order peak $q_{0}=q_{100}$, crystal grain size $L_{C}$ ), the intensity scattered by the disordered 
suspension in contact with the crystals and a backgound $I_{b g}$. For the disordered suspension, the Percus-Yevick structure factor $S_{H S}(q)$ of hard sphere with diameter $D_{H S}$ and volume fraction $\Phi_{H S}$ has been used. The two first intensities also involve the structure factor of the gold core particles FF(q).

$$
\begin{aligned}
I_{t h}(q)= & F F(q) * I_{\text {cryst }} * S_{\text {cryst }}\left(q, q_{0}, L_{C}\right) \\
& +F F(q) * I_{\text {liq }} * S_{H S}\left(q, D_{H S}, \Phi_{H S}\right)+I_{b g}
\end{aligned}
$$

Figure 5 shows a comparison between the measured radial intensity and a theoretical powder pattern for the $C_{14}$ and the $\mathrm{Z}$ structures. The $\mathrm{C} 14$ structure expected intensity corresponds quite well to the experimental pattern even if no real powder pattern could be obtained. The lattice parameter that can be deduced is $a=6.5 \mathrm{~nm}$ and the typical size of the crystalline grains built in suspensions is $0.5 \mu \mathrm{m}$. The diffraction pattern clearly does not fit with the computed intensity of the Z structure. Moreover, the intensity of the (100) peak in the hexagonal phase vanishes in the $\mathrm{Z}$ structure and this is not compatible with Figure $2 \mathrm{~b}$ where the (100) peak is clearly visible even if its intensity is low. X-Ray experimental results are thus clearly perfectly coherent with a C14 Frank and Kasper structure.

The cryo-TEM experiments do not contredict the C14 FK structure without clearly confirming it. The small grain shown in Figure 3a can be seen either as a slice of the C14 structure with thickness $c / 2$ as shown in Figure 6 a or the projection of a Z structure perpendicular to the 3-fold axis. Observing small flat grains instead of quasi-spherical ones could be due to the effect of the cryo-tem grid preparation that could disturb the soft grain shape and induce a grain flattening. However the Z-phase structure is certainly energetically very close from the C14 structure and could thus also be observed in small crystals whereas the larger crystals clearly exhibit a C14 structure as seen by x-ray scattering. The second TEM image can be interpreted by layers piling perpendicular to the (103) direction, one of the densest plane family that produces intense Bragg peaks (Figure 6b). Other projections of the C14 FK structure are shown in Supplementary Information. The emulsions are very sensitive 
to the electron beam and unfortunately cryo-tomography performed on these emulsions could not really help to confirm the structure. The future challenge is to grow mechanically stable domains that could be analysed by tomography.

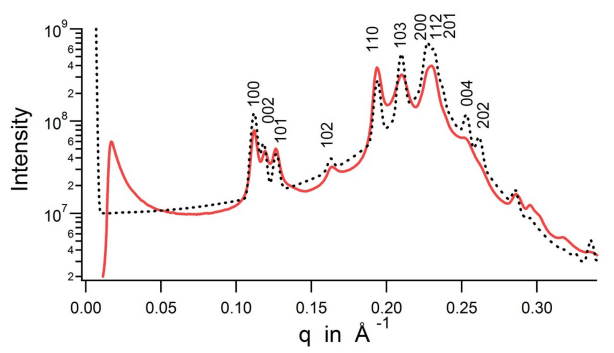

a)

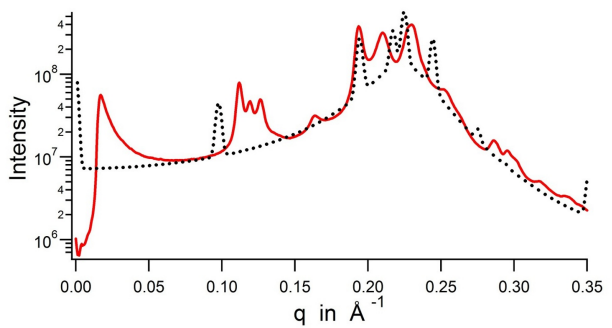

b)

Figure 5: Comparison of the measured radial intensity in suspensions (full line) with the theoretical one (dotted line) : a) for the $\mathrm{C} 14$ structure b) for the $\mathrm{Z}$ structure. The parameters used are for the crystalline contribution: $q_{0}=0.112 \AA^{-1}, L_{C} \approx 500 \mathrm{~nm}$, for the suspension contribution: $D_{H S}=3.02 \mathrm{~nm}, \Phi_{H S}=48.8 \%$. The lattice parameters are thus: $a=6.5 \mathrm{~nm}$ for both structures, $c_{C 14}=10.6 \mathrm{~nm}$ and $c_{Z}=6.45 \mathrm{~nm}$.

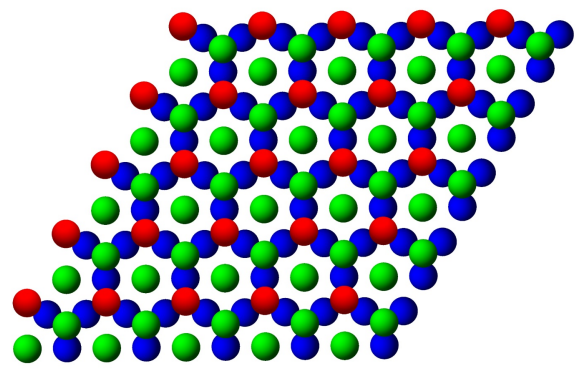

a)

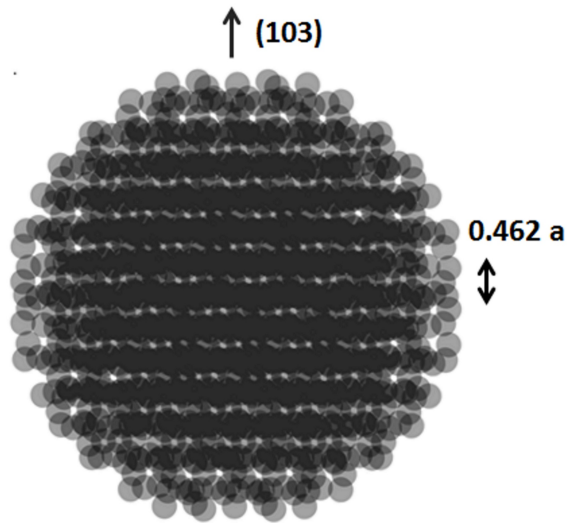

b)

Figure 6: a) A slice of the $\mathrm{C} 14$ structure with thickness $\mathrm{c} / 2$; the colors correspond to that of Figure 4a. The projection of the $\mathrm{Z}$ structure in the hexagonal plane has exactly the same structure. b) Projection of the C14 structure on a plane containing the (103) Bragg direction.

\section{Energetic approach}

Our experimental results clearly show that gold nanoparticles close to $2 \mathrm{~nm}$ diameter size covered with hexanethiol build, at room temperature, a non standard stable phase, nor FCC 
or BCC that has been interpreted as being a C14 hexagonal Frank-Kasper phase. This hexagonal phase with a large lattice parameter is observed for a ratio $\frac{2 L}{D} \approx 0.7$ that is for a ratio where a transition for which FCC towards BCC structure as been previously observed. This ratio is certainly not the only pertinent parameter. Understanding what parameters can drive the final structure of the NP superlattices is essential to control their self-assembly. For such small nanoparticles, the gold cores are not perfectly spherical but due to the presence of the ligand corona, this effect will be neglected. The parameters that are certainly involved in this problem are: the diameter $D$ of the core diameter, the extended length $L$ of the ligands, their grafting density $\sigma$, the volume occupied by each ligand v. For alkane chains wih $n=6$ carbon atoms, $L$ is equal to $0.84 \mathrm{~nm}$ and $\mathrm{v}$ is equal to $0.18 \mathrm{~nm}^{3} .{ }^{18}$ The volume occupied by each nanoparticle, when the corona is dense, is given by :

$$
V_{N P}=\frac{\pi D^{3}}{6}+\sigma \pi D^{2} \mathrm{v}=\frac{\pi D^{3}}{6}\left(1+6 \frac{L}{D}(\sigma A)\right)
$$

where $A=\frac{\mathrm{v}}{L}$ can be interpreted as the section of the chain and is roughly independant of the chain length. Let us first assume that there is no solvent still remaining in the crystal and that the soft corona are dense. The lattice parameter of a given structure is therefore deduced from: $V_{\text {cell }}=N_{\text {cell }} V_{N P}$. In each structure two geometrical parameters are important. One is the minimum distance between two neighbouring particles $d_{1}$ that must be larger than the core diameter $D=2.4 \mathrm{~nm}$. The other geometrical constraint is due to the fact that the whole volume between the gold cores must be filled by the ligand chains. Thus each point in this volume must be located at a distance from a NP surface smaller than $L$. Let $d_{2}$ be the maximum distance between any point in the structure and a nanoparticle center; $d_{2}$ is also the maximum radius of the coordination polyhedra. Thus $d_{2}$ must be smaller than $D / 2+L=2.05 \mathrm{~nm}$ to avoid empty space in the structure. Table 1 gives the typical value of all these parameters in our system for a given grafting density. The lattice parameters in the five different structures thus correspond to the same NP concentration for which the particles with a dense soft corona are expected to fill totally the space. 
Table 1: Parameters involved in the different structures with the same NP concentration corresponding to the densest packing (volume fraction equal to 1) of gold nanoparticles with dense soft corona. The NP gold core diameter is $D=2.4 \mathrm{~nm}$ and they are covered with hexanethiol with grafing density $\sigma=5.4 \mathrm{~nm}^{-2}$. $N_{\text {cell }}$ is the number of NPs in each cell, $\bar{Z}$ is the mean coordination number, $\bar{N}_{n}$ is the mean number of Voronoi polyhedron faces, $a$ is the lattice parameter in $\mathrm{nm}, d_{1}$ is the smallest distance between NPs, $d_{2}$ is the maximum distance between any point in the structure.

\begin{tabular}{l|c|c|c|c|c|c} 
Structure & $N_{\text {cell }}$ & $\bar{Z}$ & $\bar{N}_{n}$ & $a$ in $\mathrm{nm}$ & $d_{1}$ in $\mathrm{nm}$ & $d_{2}$ in $\mathrm{nm}$ \\
\hline FCC & 4 & 12 & $15^{19}$ & 4.68 & 3.25 & 2.30 \\
BCC & 2 & 8 & 14 & 3.72 & 3.16 & 2.04 \\
Fd3m=C15 & 24 & $40 / 3(13.33)$ & $40 / 3$ & 8.51 & 2.96 & 2.10 \\
$\mathrm{MgZn}_{2}=\mathrm{C} 14$ & 12 & $40 / 3$ & $40 / 3$ & 6.01 & 2.96 & 2.10 \\
$\mathrm{Zr}_{4} \mathrm{Al}_{3}=\mathrm{Z}$ & 7 & $94 / 7=13.43$ & 13.43 & 5.93 & 2.95 & 2.15
\end{tabular}

Table1 clearly shows that assuming that the nanoparticles totally fill the space rules out the FCC phase due to geometrical constraint: $d_{2}$ is too large, that means that octahedral sites are too big compared to the the chain length. This is not the case for the BCC structure. Nevertheless it is not the final structure. Geometrical parameters are also compatible with the C14, C15 and Z FK structures. The cell parameter deduced from this geometrical approach is smaller than the experimental lattice constant showing that the corona is not dense but swollen and solvent is probably still present. From a purely geometrical point of view, both BCC, C14, C15 and Z structures could be expected for dense soft corona (NP volume fraction equal to 1). FCC is allowed only if solvant is still present between the NPs.

In micellar systems the stability of the BCC structure is explained using entropic arguments. But for metallic NPs, the van der Waals energy between the gold cores cannot be ignored and is much larger than in micellar phases. The Hamaker constant of gold atoms interacting through oil is indeed very large. Its value is still under debate but $75 \mathrm{kT}$ is a value that is commonly used. ${ }^{20}$ We have computed the van der Waals energy for all the structures with the same NP concentration either for dense corona or for the observed NPs concentration derived from SAXS analysis. For both cases, the mean van der Waals energy per nanoparticle is lower for the Frank-Kasper phases. For a volume fraction equal to 1, the van der Waals energy per NP is $U_{F K 1}=-8.7 \mathrm{kT}$ for the $\mathrm{C} 15$ or $\mathrm{C} 14$ structure and $U_{B C C 1}=-7$ 
$\mathrm{kT}$ for the BCC structure and thus a difference of van der Waals energy per particle of the order of $1.7 \mathrm{kT}$ between the BCC phase and the FK phases. For the NP particle concentration experimentally observed, the van der Waals energy per NP is $U_{F K 2}=-3.59 \mathrm{kT}$ for the $\mathrm{C} 14$ or $\mathrm{C} 15$ structure, $U_{Z}=-3.50 \mathrm{kT}$ for the $\mathrm{Z}$ phase, $U_{B C C 2}=-3.47 \mathrm{kT}$ for the $\mathrm{BCC}$ phase and $U_{F C C 2}=-3.03 \mathrm{kT}$ for the FCC phase. For short ligand chains, the van der Waals energy difference is certainly large enough to dominate entropic or elastic chain free energy and this explains why Frank-Kasper phases can appear in this system. This is no longer the case for longer ligands for which the distance between the NPs will be larger and entropic effects should become significant compared to van der Waals attraction. For dense systems, the difference of the van der Waals energy per particle, with the same core diameter and covered with dodecanethiol at the same grafting density, is only $0.2 \mathrm{kT}$ between the BCC and the $\mathrm{C} 14$ structure. The remaining question is why the $\mathrm{C} 14$ structure has been observed and not the $\mathrm{C} 15$ one... These Frank-Kasper phases are very close one from the other and the difference in their energy is certainly very subtle.

\section{Conclusion}

In this paper we report the first experimental observation of a hexagonal phase with a large lattice parameter in superlattices of hydrophobically-coated gold nanoparticles at room temperature. This phase has been interpreted as a Frank-Kasper phase (C14) that is the HCP version of the Fd3m structure commonly observed in micellar systems. We show that the van der Waals attraction favors Frank-Kasper phases by comparison with the BCC or FCC structure. However no simple argument can explain why the Frank-Kasper phase that is observed is hexagonal and not cubic. Optical plasmon resonance are observed for larger gold core particles stabilized with longer ligands. For longer ligands, entropic effects have certainly to be considered and it is not clear whether larger NP could exhibit FK phases. Nevertheless this result opens a route towards building complex gold nanoparticles 
superlattices with original physical properties.

\section{Experimental}

\section{Materials}

In this study we have used small gold nanoparticles with diameter close to $2 \mathrm{~nm}$ capped with hexanethiol ligands. They have been synthetized using the Brust procedure. ${ }^{21}$ They can be suspended in various oils such as n-alkanes, cyclohexane or toluene. Organic solvents and the other chemicals were purchased from Sigma-Aldrich Co., and were used as received. The size and polydispersity of NP gold core have been determined using small angle X-ray scattering (SAXS) on dilute suspensions. The Brust synthesis is very reproducible and the typical gold core diameter is $D=2.4 \mathrm{~nm}$ (with $16 \%$ polydispersity). The grafting density, 5.4 ligands per $\mathrm{nm}^{2}$, has been determined by thermogravimetry analysis and is comparable to standard value. The length of the extended ligand chain is $\ell_{6}=0.84 \mathrm{~nm}$ and thus the maximum external diameter of the gold NPs is $D_{M}=D+2 \ell_{6}=4.1 \mathrm{~nm}$. In gold NP superlattices, the interpenetration of the ligand chains is commonly observed. ${ }^{22}$ A large repulsion between the ligands is predicted when they are fully interpenetrated and the corresponding distance between the spheres is $D_{P}=D+\ell_{6}=3.24 \mathrm{~nm}$. For hard sphere with diameter $D_{M}=4 \mathrm{~nm}$

(resp: $D_{P}=3.2 \mathrm{~nm}$ ), the first peak position for classical structures is $0.192 \AA^{-1}$ (resp. 0.24 $\AA^{-1}$ ) for FCC and BCC and $0.181 \AA^{-1}$ (resp. $0.226 \AA^{-1}$ ) for HCP.

\section{Methods}

NPs are first suspended in various solvents either volatile solvents (cyclohexane, heptane, toluene) or mixtures of non volatile alkanes (n-dodecane, hexadecane) with a volatile solvent. The initial NP volume fraction is typically less than $5 \%$ (or 30w\%) for which no self-assembly in superlattices occurs. The suspensions are poured in flat glass capillaries (VitroCom, USA) that are sealed only at one end. The capillaries are left open at room temperature. Due to the 
evaporation, the NP concentration in the capillary slowly increases and is followed measuring the x-ray absorbtion as well as weight measurements. The second end of the capillary is sealed when the wished NP concentration is reached. Using flat capillaries has several advantages. Their thickness $(0.1 \mathrm{~mm})$ is well controlled, that is important to determine precisely the NP concentration by X-ray absorbtion. Their flat surfaces can promote the nucleation and growth of monodomains. Oil in water emulsions have also been used. ${ }^{23}$ Droplets of NP suspension in toluene $(5 \mathrm{w} \%)$ are dispersed in water with the help of a non ionic surfactant (Pluronic P123 from BASF). Ostwald ripening promotes a spontaneous increase of the NP concentration in the smaller droplets and therefore their self-assembly.

The superlattice structures are determined by SAXS and the experiments have been performed on the Swing beamline of the Soleil synchrotron laboratory (Gif/Yvette, France) and on home set-ups (LPS, Orsay, France). Complementary tests have been done on the D2AM beamline of the ESRF synchrotron laboratory (Grenoble, France). All experiments have been performed at room temperature. The patterns commonly observed are mainly textured patterns produced by several single domains as in 2a. Single domain patterns as that of $2 \mathrm{~b}$ are rarely observed.

Superlattice grains built in emulsion have also been analysed by cryogenic transmission electron microscopy (cryo-TEM). A $3 \mu l$ drop of the sample was deposited onto a glowdischarged Quantifoil R2/2 grid (Quantifoil Micro Tools, Jena, Germany) and vitrified in liquid ethane using a home-made cryo-fixation device. The cryo-specimens were transferred to a Gatan 626 cryo-holder (Gatan Inc., Warrendale, PA, USA) and examined at $-180^{\circ} \mathrm{C}$ with a JEM 2010 Field Emission Gun cryo-Transmission Electron Microscope (Jeol, Tokyo, Japan) using an acceleration voltage of $200 \mathrm{kV}$ and a $50000 \mathrm{x}$ magnification. Images were recorded with a Gatan $4 \mathrm{~K}$ x $4 \mathrm{~K}$ Ultrascan camera with 1 to $1.5 \mu \mathrm{m}$ of defocus under lowelectron-dose conditions. 


\section{Acknowledgement}

The authors thank F. Meneau (Soleil), C. Rochas (ESRF), O. Taché (MOMAC experiment, CEA-LIONS+LPS, funded by RTRA "Triangle de la Physique") for their efficient and kind assistance for collecting the x-ray data. For the cryo-TEM experiments, the authors acknowledge the friendly help of J. Degrouard and the financial support from the french CNRS (FR3507) and CEA METSA network (www.metsa.fr). The authors have benefited from fruitful discussions with M. Impéror-Clerc and J. Schmitt (LPS, Orsay).

\section{Supporting Information Available}

Geometric features of the $\mathrm{C} 14$ and Z phases. Bragg peak positions and intensities for C14, $\mathrm{C} 15$ and $\mathrm{Z}$ phases. Projection of the $\mathrm{C} 14$ structure along various directions. This material is available free of charge via the Internet at http://pubs.acs.org/.

\section{References}

1. Dzugutov, M. Formation of a dodecagonal quasicrystalline phase in a simple monatomic liquid. Phys. Rev. Lett. 1993, 70, 2924-2927.

2. Damasceno, P. F.; Engel, M.; Glotzer, S. C. Predictive Self-Assembly of Polyhedra into Complex Structures. Science 2012, 337, 453-457.

3. Frank, F. C.; Kasper, J. S. Complex alloy structures regarded as sphere packings. I. Definitions and basic principles. Acta Crystallogr. 1958, 11, 184-190.

4. Shechtman, D.; Blech, I.; Gratias, D.; Cahn, J. W. Metallic phase with long-range orientational order and no translational symmetry. Phys. Rev. Lett. 1984, 53, 19511953.

5. Ungar, G.; Zeng, X. Frank-Kasper, quasicrystalline and related phases in liquid crystals. Soft Matter 2005, 1, 95-106. 
6. Talapin, D. V.; Shevchenko, E. V.; Bodnarchuk1, M. I.; Ye, X.; Chen, J.; Murray, C. B. Quasicrystalline order in self-assembled binary nanoparticle superlattices. Nature 2009, 461, 964-967.

7. Ziherl, P.; Kamien, R. D. Maximizing entropy by minimizing area:? Towards a new principle of self-organization. J. Phys. Chem. B 2001, 105, 10147-10158.

8. Iacovella, C. R.; Keys, A. S.; Glotzer, S. C. Self-assembly of soft-matter quasicrystals and their approximants. Proc. Natl. Acad. Sci. U. S. A. 2011, 108, 20935-20940.

9. Seddon, J.; Templer, R. In Handbook of Biological Physics: Structure and Dynamics of Membranes; Lipowsky, R., Sackmann, E., Eds.; Elsevier Science, 1995; pp 97-160.

10. Ungar, G.; Liu, Y.; Zeng, X.; Percec, V.; Cho, W.-D. Giant supramolecular liquid crystal lattice. Science 2003, 299, 1208-1211.

11. Lee, S.; Bluemle, M. J.; Bates, F. S. Discovery of a Frank-Kasper phase in sphere-forming block copolymer melts. Science 2010, 330, 349-353.

12. Sangwoo, L.; Leighton, C.; Bates, F. S. Sphericity and symmetry breaking in the formation of Frank-Kasper phases from one component materials. Proc. Natl. Acad. Sci. U. S. A. 2014, 111, 17723-17731.

13. Goodfellow, B. W.; Rasch, M. R.; Hessel, C. M.; Patel, R. N.; Smilgies, D.-M.; Korgel, B. A. Ordered structure rearrangements in heated gold nanocrystal superlattices. Nano Lett. 2013, 13, 5710-5714.

14. Laves, F. In Crystal Chemistry: Structure of Metals, Metalloids and their Compounds.; Book, C. J. S. M. R., Ed.; London: Butterworth, 1949.

15. Landman, U.; Luedtke, W. D. Small is different: energetic, structural, thermal, and mechanical properties of passivated nanocluster assemblies. Faraday Discuss. 2004, 125, $1-22$. 
16. Whetten, R. L.; Shafigullin, M. N.; Khoury, J. T.; Schaaff, T. G.; Vezmar, I.; Alvarez, M. M.; Wilkinson, A. Crystal Structures of Molecular Gold Nanocrystal Arrays. Acc. Chem. Res. 1999, 32, 397-406.

17. Wang, Z.; Schliehe, C.; Bian, K.; Dale, D.; Bassett, W. A.; Hanrath, T.; Klinke, C.; Weller, H. Correlating Superlattice Polymorphs to Internanoparticle Distance, Packing Density, and Surface Lattice in Assemblies of PbS Nanoparticles. Nano Lett. 2013, 13, $1303-1311$.

18. Israelachvili, J. N., Ed. Intermolecular and Surface Forces, 3rd ed.; Academic Press: San Diego, 2011.

19. Lucarini, V. Three-Dimensional Random Voronoi Tessellations: From Cubic Crustal Lattices to Poisson Point Processes. J. Stat. Phys. 2009, 134, 185-206.

20. Leff, D. V.; Ohara, P. C.; Heath, J. R.; Gelbart, W. M. Thermodynamic Control of Gold Nanocrystal Size: Experiment and Theory. J. Phys. Chem. 1995, 99, 7036-7041.

21. Brust, M.; Fink, J.; Bethell, D.; Schiffrin, D. J.; Kiely, C. Synthesis and reactions of functionalised gold nanoparticles. J. Chem. Soc., Chem. Commun. 1995, 1655-1656.

22. Daniel, M. C.; Astruc, D. Gold Nanoparticles: Assembly, Supramolecular Chemistry, Quantum-Size-Related Properties, and Applications toward Biology, Catalysis, and Nanotechnology. Chem. Rev. 2004, 104, 293-346.

23. Lacava, J.; Ouali, A.-A.; Raillard, B.; Kraus, T. On the behaviour of nanoparticles in oil-in-water emulsions with different surfactants. Soft Matter 2014, 10, 1696-1704. 
Graphical TOC Entry

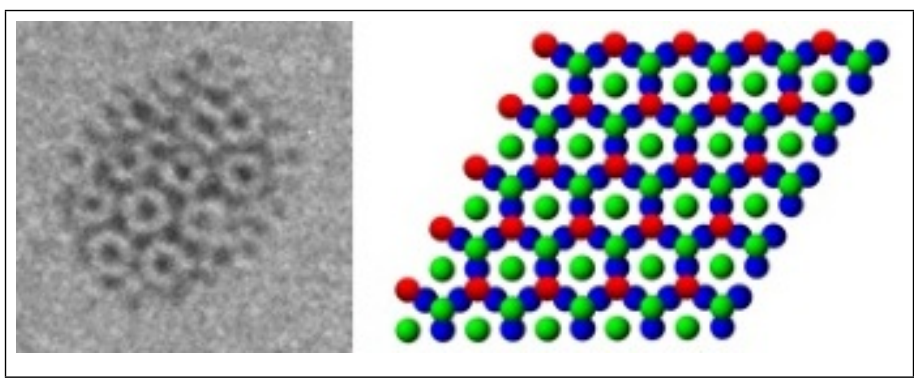

